\title{
Assessing advantages of sequential boron neutron capture therapy (BNCT) in an oral cancer model with normalized blood vessels
}

\author{
ANA J. MOLINARI ${ }^{1}$, SILVIA I. THORP ${ }^{2}$, AGUSTINA M. PORTU ${ }^{1,3}$, \\ GISELA SAINT MARTIN ${ }^{1}$, EMILIANO C. C. POZZI ${ }^{1,4}$, ELISA M. HEBER ${ }^{1}$, \\ SILVA BORTOLUSSI ${ }^{5}$, MARIA E. ITOIZ ${ }^{6}$, ROMINA F. AROMANDO ${ }^{6}$, \\ ANDREA MONTI HUGHES ${ }^{1}$, MARCELA A. GARABALINO ${ }^{1}$, SAVERIO ALTIERI ${ }^{5}$, \\ VERÓNICA A. TRIVILLIN ${ }^{1,3}$ \& AMANDA E. SCHWINT ${ }^{1,3}$ \\ ${ }^{1}$ Department of Radiobiology, National Atomic Energy Commission (CNEA), San Martin, Province Buenos Aires, \\ Argentina, ${ }^{2}$ Department of Instrumentation and Control, CNEA, San Martin, Province Buenos Aires, \\ Argentina, ${ }^{3}$ National Research Council (CONICET), Ciudad Autónoma de Buenos Aires, Argentina, ${ }^{4}$ Department of \\ Research and Production Reactors, CNEA, San Martin, Province Buenos Aires, Argentina, ${ }^{5}$ Department of Nuclear \\ and Theoretical Physics, University of Pavia, Pavia, Italy, and ${ }^{6}$ Faculty of Dentistry, University of Buenos Aires, \\ Buenos Aires, Argentina
}

\section{ABSTRACT}

Background. We previously demonstrated the therapeutic success of sequential boron neutron capture therapy (SeqBNCT) in the hamster cheek pouch oral cancer model. It consists of BPA-BNCT followed by GB-10-BNCT 24 or 48 hours later. Additionally, we proved that tumor blood vessel normalization with thalidomide prior to BPA-BNCT improves tumor control. The aim of the present study was to evaluate the therapeutic efficacy and explore potential boron microdistribution changes in Seq-BNCT preceded by tumor blood vessel normalization.

Material and Methods. Tumor bearing animals were treated with thalidomide for tumor blood vessel normalization, followed by Seq-BNCT $(\mathrm{Th}+\mathrm{Seq}-\mathrm{BNCT})$ or Seq-Beam Only $(\mathrm{Th}+\mathrm{Seq}-\mathrm{BO})$ in the window of normalization. Boron microdistribution was assessed by neutron autoradiography.

Results. Th+ Seq-BNCT induced overall tumor response of 100\%, with 87 (4)\% complete tumor response. No cases of severe mucositis in dose-limiting precancerous tissue were observed. Differences in boron homogeneity between tumors pre-treated and not pre-treated with thalidomide were observed.

Conclusion. Th + Seq-BNCT achieved, for the first time, response in all treated tumors. Increased homogeneity in tumor boron microdistribution is associated to an improvement in tumor control.

Boron neutron capture therapy (BNCT) is a binary treatment modality that combines irradiation with a thermal or epithermal neutron beam with tumorseeking, boron-containing drugs that are taken up preferentially by neoplastic cells to produce selective irradiation of tumor tissue. The high linear energy transfer (LET) alpha particles and recoiling ${ }^{7} \mathrm{Li}$ nuclei emitted during the ${ }^{10} \mathrm{~B}(\mathrm{n}, \alpha){ }^{7} \mathrm{Li}$ reaction in tissue are known to have a high relative biological effectiveness (RBE). Their short path length in tissue $(6-10 \mu \mathrm{m})$ limits their effect mostly to cells containing ${ }^{10} \mathrm{~B}$ atoms, providing a strategy to dam- age tumor cells while protecting healthy tissue within the treatment volume [1].

Clinical interest in BNCT has focused primarily on high-grade gliomas, and more recently on patients with recurrent tumors of the head and neck region who have failed conventional therapy $[2,3]$. To date, the clinical results have demonstrated the safety and therapeutic potential of this technique. Despite recent progress in optimizing BNCT for different pathologies [e.g. 4], there is undoubtedly room for improvement in terms of enhancing tumor response at no cost in terms of toxicity.

Correspondence: A. E. Schwint, Head Radiation Pathology Division, Department of Radiobiology, National Atomic Energy Commission, Avenida General Paz 1499, B1650KNA San Martin, Province Buenos Aires, Argentina. Tel: +54 116772 7149. Fax: +54 11 6772 7188. E-mail: schwint@cnea.gov.ar 
Much effort has been expended to search for the 'ideal' ${ }^{10} \mathrm{~B}$ compound that would potentially replace the three compounds currently authorized for use in human beings, boronophenylalanine (BPA) and sodium borocaptate $(\mathrm{BSH})$, used in clinical trials, and decahydrodecaborate (GB-10). Based on the relative non-selectivity of the two drugs that currently are in clinical use, the development of new, more selective boron delivery agents probably is the single greatest need for future progress of BNCT [5].

The requirements of boron carriers for BNCT have been well established. Boron compounds are traditionally required to be non-toxic at therapeutic doses, to reach tumor:blood and tumor:normal tissue boron concentration ratios of approximately 3:1 and to deliver enough boron atoms to tumor so that the number of capture reactions that occur are enough to induce cell death [e.g. 5,6]. Although the importance of determining the spatial localization of the boron compounds under study has been pointed out [e.g. 7], to date, homogeneous tumor targeting has not been specifically established as a requirement of the 'ideal' boron carrier. The fact that cells poorly loaded with boron will be refractory to treatment, posing a risk of recurrence, makes homogeneous tumor boron targeting pivotal to the success of BNCT. Within this context, in addition to analytical techniques for the measurement of gross boron concentration, complementary methods to determine boron microdistribution, such as neutron autoradiography will be necessary [e.g. 8-10].

Seeking to improve the therapeutic efficacy of BNCT without enhancing mucositis in dose-limiting tissue, an issue of great clinical relevance [e.g. 3], we previously proposed sequential BNCT (Seq-BNCT) for the treatment of oral cancer in the hamster cheek pouch model. Seq-BNCT is based on the application of BNCT mediated by BPA (BPA-BNCT) followed by BNCT mediated by GB-10 (GB-10-BNCT), with an interval between irradiations of 24 or 48 hours [11].

Further striving to optimize BNCT for the treatment of oral cancer in the hamster model, and given that boron targeting of the largest possible proportion of tumor cells contributes to the success of BNCT, we developed a technique to normalize aberrant blood vessels in the hamster cheek pouch oral cancer model. We validated this technique by measuring significant reductions in aberrant tumor vascular hyperpermeability and compressive forces [12]. The abnormal structure and function of tumor blood vessels compromise blood flow and hinder effective convective fluid transport, resulting in the impaired distribution of blood-borne therapeutic agents in general. Tumor blood vessel normalization would lead to less leaky, less dilated and less tortuous vessels, decreased interstitial fluid pressure, increased tumor oxygenation and improved penetration of drugs in tumors [13]. Blood vessel normalization prior to BPA-BNCT significantly increased overall tumor response and reduced mucositis in precancerous tissue [14].

Within this context, the aim of the present study was to assess the therapeutic efficacy (tumor response and toxicity) and potential changes in boron microdistribution of both therapeutic modalities combined, i.e. blood vessel normalization prior to Seq-BNCT, in the hamster cheek pouch oral cancer model. The working hypothesis was that by combining both treatment modalities we would favor homogeneous distribution of the boron compounds in tumor and improve tumor response, without enhancing dose-limiting mucositis in precancerous tissue. To test our working hypothesis, we assessed outcome in terms of tumor response and potential toxicity, examined potential changes in the microdistribution of boron associated to the treatment protocol and analyzed their potential role in outcome. The knowledge of BNCT radiobiology derived from this study will serve to optimize treatment protocols and will also be applicable in the future to potentially 'more perfect' ${ }^{10} \mathrm{~B}$ compounds.

\section{Material and methods}

\section{Tumor induction}

The right cheek pouch of Syrian hamsters received topical application of $0.5 \%$ dimethyl-1,2-benzanthracene (DMBA) in mineral oil twice a week for approximately 12 weeks as previously described [11]. Guidelines of the Committee for Laboratory Animal Care and Use of the National Atomic Energy Commission were followed throughout.

\section{In vivo BNCT studies}

Irradiations were performed as previously described [e.g. 11] at the biomedical facility of the RA-3 research and production nuclear reactor constructed by the National Atomic Energy Commission in Buenos Aires [15]. A tunnel penetrating the graphite structure of the thermal column enables the insertion of samples into a near-isotropic neutron field while the reactor is in normal operation. The neutron field is very well thermalized, making the radiation dose component from hydrogen recoil (i.e. fast neutron dose) in tissue negligible. A shield was constructed to protect the body of the animal from the thermal neutron flux while exposing the everted cheek pouch bearing tumors. The enclosure was fabricated from 
plates composed of a 6-mm layer of lithium carbonate enriched to $95 \%$ in lithium-6, sealed within sheets of Lucite. The hamster pouch was everted out of the enclosure onto a protruding shelf. The temperature at the irradiation site within the tunnel was approximately $30^{\circ} \mathrm{C}$. Physical dosimetry data corresponding to the irradiation system have been previously reported [16]. Briefly, in the irradiation position, the thermal neutron flux was $7.0 \pm 0.1 \times 10^{9} \mathrm{n} / \mathrm{cm}^{2} / \mathrm{s}$ and the dose rate of gamma rays in air was $6.6 \pm 0.6 \mathrm{~Gy} / \mathrm{h}$.

One group of hamsters $(\mathrm{n}=8)$ bearing a total of 78 tumors (Th+Seq-BNCT) was treated with thalidomide as previously described $[12,14]$. Briefly, the animals were treated with two doses of $200 \mathrm{mg}$ thalidomide/kg body weight in dimethyl sulfoxide (DMSO) $(112 \mathrm{mg}$ thalidomide $/ \mathrm{ml}$ DMSO) intraperitoneally (i.p.) into tumor-bearing hamsters on two consecutive days. Thalidomide was a generous gift of Triquim S.A. (Argentina). In the window of normalization the animals were treated with Seq-BNCT, i.e. BPA-BNCT followed by GB-10-BNCT 48 hours later at a total absorbed dose prescribed to tumor of 9.9 Gy as previously described [11]. The actual absorbed dose may differ from the prescribed absorbed dose due to variations in boron content that cannot be monitored online. BPA $(0.14 \mathrm{M})$ was administered i.p. at a dose of $15.5 \mathrm{mg}{ }^{10} \mathrm{~B} / \mathrm{kg}$ body weight and GB-10 was administered intravenously (i.v.) at a dose of $50 \mathrm{mg}{ }^{10} \mathrm{~B} / \mathrm{kg}$ body weight as described in Molinari et al. [11]. A second group of hamsters $(n=4)$ bearing a total of 33 tumors was treated with thalidomide as described above and then treated with sequential beam-only (BO) irradiations 48 hours apart $(\mathrm{Th}+\mathrm{Seq}-\mathrm{BO}) . \mathrm{Th}+\mathrm{Seq}-\mathrm{BO}$ irradiations were performed at the same neutron fluence as $\mathrm{Th}+$ Seq-BNCT irradiations to assess the effect of background dose. For ethical reasons it is not possible to duplicate experiments with animals if the results have already been published. Hence, the data for the control groups were taken from a previous study [11]. To be able to compare the outcome of the $\mathrm{Th}+$ Seq-BNCT and $\mathrm{Th}+\mathrm{Seq}-\mathrm{BO}$ groups (this study) with the outcome of the SeqBNCT group with no prior thalidomide treatment (Th-Seq-BNCT) and the BO group without prior blood vessel normalization (Th-Seq-BO) [10], the present irradiations were performed at the same neutron fluence as those in the previous study.

\section{Follow-up}

The tumor and precancerous tissue responses were assessed by visual inspection and tumor volume assays pre-treatment, and at 2, 7, 14, 21 and 28 days post-treatment as previously described [e.g. 11]. A reduction from initial tumor volume was considered as partial response (PR). Complete tumor response (CR) was defined as disappearance of the tumor on visual inspection and no evidence of tumor on histological analysis when assessment was possible. Overall response (OR) was defined as PR+CR. To evaluate toxicity, clinical signs and body weight of all the animals were monitored regularly. The severity of mucositis was evaluated semi-quantitatively in precancerous tissue and normal pouch tissue according to a previously described [e.g. 11,14] oral mucositis six-grade scale based on macroscopic features, adapted for the carcinogen-treated hamster cheek pouch, from the WHO classification for oral mucositis in human subjects [17] and from the sixpoint grading system for normal hamster cheek pouches [18].

At the last time-point evaluated the animals were euthanized for histological analysis of persistent tumors and precancerous tissue surrounding treated tumors.

\section{Boron concentration and microdistribution}

We performed biodistribution studies to determine potential changes, induced by pre-treatment with thalidomide, on BPA gross uptake [14] and microdistribution in tumor (this study). Likewise, we assessed potential changes induced by pre-treatment with thalidomide followed by BPA-BNCT, on gross boron concentration and boron microdistribution in tumor, precancerous and normal pouch tissue of animals injected with GB-10 as in SeqBNCT. Four experimental groups of tumor bearing hamsters were evaluated: $\mathrm{Th}+\mathrm{BPA}$ (animals treated with two doses of thalidomide at the same dose described in section in vivo BNCT studies. In the window of normalization the animals were injected with BPA $15.5 \mathrm{mg}{ }^{10} \mathrm{~B} / \mathrm{kg}$ body weight and tissue samples were excised three hours after boron compound administration); Th-BPA (the same as $\mathrm{Th}+\mathrm{BPA}$ but with no prior thalidomide treatment); Th + GB-10 48 hours after BPA-BNCT (animals treated with two doses of thalidomide as Th+ BPA. In the window of normalization the animals were treated with BPA-BNCT and 48 hours after the irradiation with BPA animals were injected with GB-10 $50 \mathrm{mg}{ }^{10} \mathrm{~B} / \mathrm{kg}$ body weight. Tissue samples were excised three hours after GB-10 administration); Th-GB-10 48 hours after BPA-BNCT (the same as Th+ GB-10 48 hours after BPA-BNCT but with no prior thalidomide treatment). As previously described, gross boron concentration was assessed by inductively coupled plasma mass spectrometry (ICP-MS) [14]. Boron microdistribution was analyzed by neutron qualitative autoradiography (QLA) 
$[8,10]$ and quantitative autoradiography (QTA) [9] Briefly, all samples were fixed with liquid nitrogen, sectioned in a cryostat and mounted on polycarbonate nuclear track detector foils. Three animals were assessed for each experimental group, and one sample of tumor, premalignant and normal pouch tissue was excised for each animal. Three sections per tissue sample were analyzed. Samples for QTA were irradiated with $10^{12} \mathrm{n} / \mathrm{cm}^{2}$ and samples for QLA were irradiated with $10^{13} \mathrm{n} / \mathrm{cm}^{2}$ at the RA-3 nuclear reactor. QTA foils were marked with reference points, in order to assess zones of parenchyma (P) and stroma (S) for tumor samples. Track density was measured for each tumor region and then converted to ${ }^{10} \mathrm{~B}$ concentration values using a calibration system. A mean value was obtained for each area of tumor ( $\mathrm{P}$ and $\mathrm{S}$ ) whenever it was possible to unequivocally distinguish these areas within the tissue section under evaluation. The $\mathrm{P} / \mathrm{S}$ ratio was defined as an indicator of boron targeting homogeneity in tumor. A ratio of 1 indicates the highest grade of homogeneity. Results are presented as mean value \pm SD.

\section{Statistical analysis for in vivo BNCT studies}

To reduce the level of non-treatment variation, a uniform, well-mixed population of animals was chosen, in order to separate the effect of the different treatments from other contributory factors (e.g. weight, sex) which may seriously influence response. In this way, it is possible to improve the sensitivity of the statistical analysis to treatment differences, although inevitably reducing the scope of the statistical conclusions. Individuals were not distinguished from each other further to the assigned treatment. Subjects used for sampling were independently assigned between treatments, considering random selection of individuals that have been prepared under strictly the same cancerization protocol. Research was conducted under a two-strata experimental design. The first stratum level comprised BO irradiated animals, with or without thalidomide treatment, and the second stratum level was designed to compare subjects treated with Seq-BNCT, with or without thalidomide administration. To this purpose, $\mathrm{BO}$ individuals were irradiated with the same total neutron fluence as those treated with SeqBNCT, thus fulfilling the statistical conditions for a two-strata study.

Treatment was applied only in the second stratum and the type of response was categorical. Since the research relates to proportions or success rates, the aim was to study differences in response. Therefore, additivity of effects from each stratum was assumed throughout. The multinomial model (i.e. a repetitive experimental process in which there are more than two possible categories of response) was assumed to be valid for assessing success rates for tumor control. Throughout the study, the requirements for valid application of the model were assumed to hold, i.e. 1) the response of one tumor is not influenced by the responses of other tumors; 2) the probability of response is constant for a given treatment; and 3) an objective method of sample selection was employed. There is no standard statistic to apply in the comparison of models and data for the multinomial model. Since the test consists of comparing contingency tables, the raw data were directly used. Assuming that the multinomial model describes the data, then for each category $\mathrm{k}, S E_{k}=\sqrt{p_{k}\left(1-p_{k}\right) / n}$ was the estimator of the standard deviation of the mean, or standard error, where $p_{k}=k / n$ was the estimator of the proportion. In this study we abbreviated it as SE and it is represented in round brackets. For extreme values ( 0 or 1 ) of $p_{k}=k / n$, the estimator $\mathrm{SE}$ was not suitable for estimating the true standard deviation, and it was therefore neither calculated nor reported.

Under the considerations stated above, the null hypothesis to be examined in this work was that no differences in response are produced by introducing thalidomide as a modifier. The primary statistics used was the Pearson $\chi^{2}$ statistic:

$$
\mathrm{X}_{p}^{2}=\sum_{i=1}^{g} \sum_{j=1}^{c} \frac{\left(y_{i j}-\hat{y}_{i j}\right)^{2}}{\hat{y}_{i j}}
$$

where $y_{i j}$ were the observed frequencies and $\hat{y}_{i j}$ were the fitted values calculated under the proposed model. The values calculated for each statistic were referred to as $X_{0}^{2}$. The p-value $p_{0}$ is obtained by calculating:

$$
p_{0}=\operatorname{Pr}\left(\mathrm{X}^{2}(d) \geq X_{0}^{2}\right)
$$

where $d$ was the number of degrees of freedom. Statistical significance was set at $\mathrm{p}=0.05$.

\section{Statistical analysis for boron concentration and boron microdistribution}

The statistical significance of the differences in gross boron content and the $\mathrm{P} / \mathrm{S}$ values were evaluated by Student's t test. Statistical significance was set at $\mathrm{p}=0.05$.

\section{Results}

Tumor response was evaluated at 28 days posttreatment considering three arbitrary tumor sizes (small: $<10 \mathrm{~mm}^{3}$, medium: $10-100 \mathrm{~mm}^{3}$, large: $>100 \mathrm{~mm}^{3}$ ) defined to categorize tumor size at the time of irradiation and evaluate potential differential 
Table I. Tumor response [\% (SE)].

\begin{tabular}{lrlccc}
\hline Tumors & $\mathrm{n}$ & $\mathrm{CR}$ & $\mathrm{PR}$ & No response & OR \\
\hline Th-Seq-BNCT* & & & & & \\
Total & 92 & $68(5)$ & $23(5)$ & $9(3)$ & $91(3)$ \\
Large $\left(>100 \mathrm{~mm}^{3}\right)$ & 8 & $25(15)$ & $63(17)$ & $12(17)$ & $88(11)$ \\
Medium $\left(10-100 \mathrm{~mm}^{3}\right)$ & 21 & $52(11)$ & $43(11)$ & $5(6)$ & $95(5)$ \\
Small $\left(<10 \mathrm{~mm}^{3}\right)$ & 63 & $79(5)$ & $11(4)$ & $10(4)$ & $90(4)$ \\
Th+ Seq-BNCT & & & & & \\
Total & 38 & $87(4)$ & $13(4)$ & 0 & 100 \\
Large $\left(>100 \mathrm{~mm}^{3}\right)$ & 3 & 0 & 100 & 0 & 100 \\
Medium $\left(10-100 \mathrm{~mm}^{3}\right)$ & 20 & $75(10)$ & $25(10)$ & 0 & 100 \\
Small $\left(<10 \mathrm{~mm}^{3}\right)$ & 55 & $96(3)$ & $4(3)$ & 0 & 100 \\
Th-Seq-BO* & 31 & 0 & $6(4)$ & $94(4)$ & $6(4)$ \\
Total & 1 & 0 & 0 & 100 & 0 \\
Large $\left(>100 \mathrm{~mm}^{3}\right)$ & 4 & 0 & $25(22)$ & $75(22)$ & $25(22)$ \\
Medium $\left(10-100 \mathrm{~mm}^{3}\right)$ & 26 & 0 & $4(4)$ & $96(4)$ & $4(4)$ \\
Small $\left(<10 \mathrm{~mm}^{3}\right)$ & & & & & \\
Th+ Seq-BO & 33 & 0 & 0 & $94(4)$ & $6(4)$ \\
Total & 0 & 0 & 0 & 0 & 0 \\
Large $\left(>100 \mathrm{~mm}^{3}\right)$ & 8 & 0 & $4(4)$ & $96(4)$ & $4(4)$ \\
Medium $\left(10-100 \mathrm{~mm}^{3}\right)$ & 25 & & & &
\end{tabular}

*Data reported in Molinari et al., 2011.

response according to size as previously described [e.g. 11]. Table I presents tumor response for groups $\mathrm{Th}+$ Seq-BNCT and Th + Seq-BO. As control groups, and for comparative purposes, previously reported [11] tumor response data for Th-Seq-BNCT and Th-Seq-BO have been included. Although the overall tumor response (OR) for all tumor sizes taken together was remarkably high for Th-Seq-BNCT [91 (3)\%] [11], pre-treatment with thalidomide increased OR significantly to $100 \%(p=0.008)$. Likewise, pre-treatment with thalidomide significantly increased complete tumor response (CR) from $68(5) \%$ to $87(4) \%(\mathrm{p}=0.004)$.

Mucositis in precancerous tissue surrounding treated tumors (non-shielded pouch) peaked at 7-14 days after Seq-BNCT regardless of pre-treatment with thalidomide. The incidence of Grade 3 mucositis was similar for both groups, $38 \pm 17 \%$ for $\mathrm{Th}+$ Seq-BNCT (this study) versus $33 \pm 16 \%$ for Th-Seq-BNCT [11]. However, while the Th-SeqBNCT group exhibited an incidence of $22 \pm 14 \%$ Grade 4 mucositis, none of the animals in the $\mathrm{Th}+$ Seq-BNCT group exhibited Grade 4 mucositis. The animals in the Th-Seq-BO group reached Grade 2 mucositis whereas the animals in the $\mathrm{Th}+\mathrm{Seq}-\mathrm{BO}$ group did not exceed Grade 1. In all cases, mucositis reverted within the study period of 28 days and none of the normal pouches (not cancerized and shielded pouches) exceeded grade 0 of mucositis.

A light microscopy assessment of the pouch areas where a tumor had been macroscopically identified pre-treatment, and was no longer identifiable post-treatment, showed no evidence of tumor cells. Twenty-eight days post-treatment, the epithelium corresponding to the tumor site only exhibited some areas of hyperplasia (Figure 1A). The presence of fibro-hyaline tissue underlying the epithelium, embedded in granulation tissue, gave the area a scar-like appearance (Figure 1B).

No normal tissue radiotoxicity was observed with any of the protocols. No contributory changes were observed in the health status or body weight of the treated animals.

Previous biodistribution studies with BPA showed that pre-treatment to normalize tumor blood vessels did not significantly change the gross boron concentration in tumor as measured by ICP-MS [14]. Herein we assessed potential changes induced in the microlocalization of BPA. In tumor, QLA analysis revealed that BPA distribution was heterogeneous, with preferential accumulation in parenchyma and that pre-treatment with thalidomide induced a more homogeneous distribution of the boron compound (Figure 2). The parenchyma/

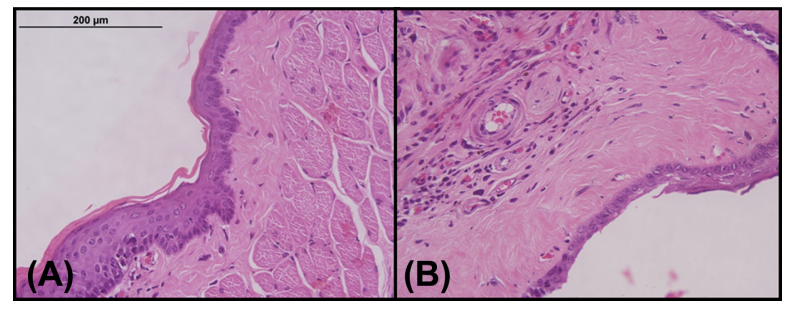

Figure 1. Light microscopy images (20X). Histological sections $(10 \mu \mathrm{m})$ of a pouch treated with $\mathrm{Th}+\mathrm{Seq}-\mathrm{BNCT}, 28$ days post-treatment (hematoxylin-eosin stain). (A) Normal-looking epithelium, with areas of hyperplasia; (B) Area with abundant fibrous hyaline tissue, with a scar-like appearance, underlying the epithelium. 


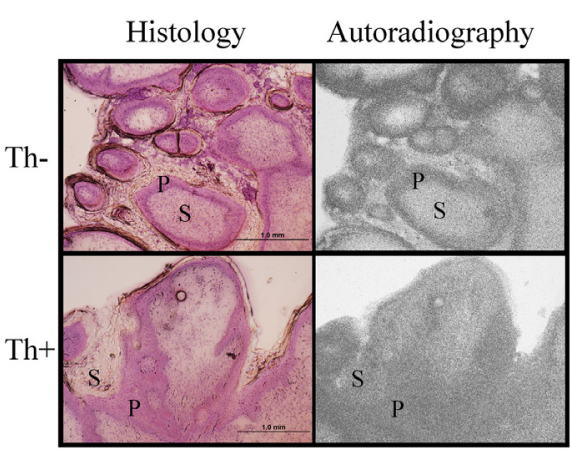

BPA

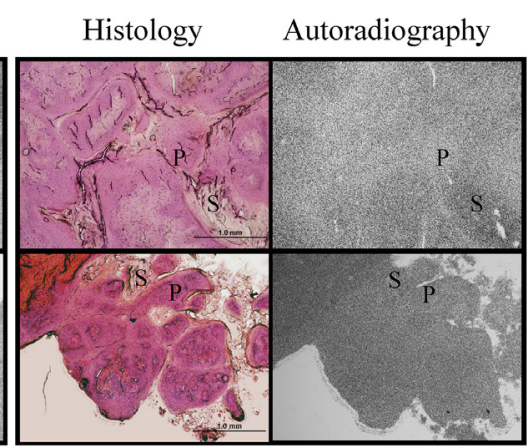

GB-10 48 hours after BPA-BNCT

Figure 2. Light microscopy images of cryostat sections $(30 \mu \mathrm{m})$ of tumor stained with hematoxylin-eosin and the corresponding neutron autoradiography images $(10 \times) . \mathrm{P}=$ parenchyma, $\mathrm{S}=$ stroma.

stroma (P/S) boron ratio was determined by QTA and used as an indicator of boron targeting homogeneity. A P/S ratio of 1 would indicate the highest degree of homogeneity. The P/S ratio was $2.1 \pm 0.4$ for Th-BPA while it was $1.6 \pm 0.2$ for $\mathrm{Th}+\mathrm{BPA}$. However, this difference did not reach statistical significance $(p=0.1846)$. Furthermore, the biodistribution studies performed herein revealed that pretreatment with thalidomide and BPA-BNCT did not alter the gross boron concentration as measured by ICP-MS in animals injected with GB-10 (27 \pm 4 ppm for animals injected with GB-10 after treatment with thalidomide and BPA-BNCT versus $32 \pm 21 \mathrm{ppm}$ for animals injected with GB-10 with no prior treatment). However, the distribution of GB-10 in Th+ GB-10 48 hours after BPA-BNCT tumors was markedly homogeneous (P/S ratio $1.0 \pm 0.2$ ), whereas it was more heterogeneous in Th-GB-10 48 hours after BPA-BNCT tumors (P/S ratio $0.74 \pm 0.04$ ) (Figure 2). This difference in boron homogeneity between tumors pre-treated and

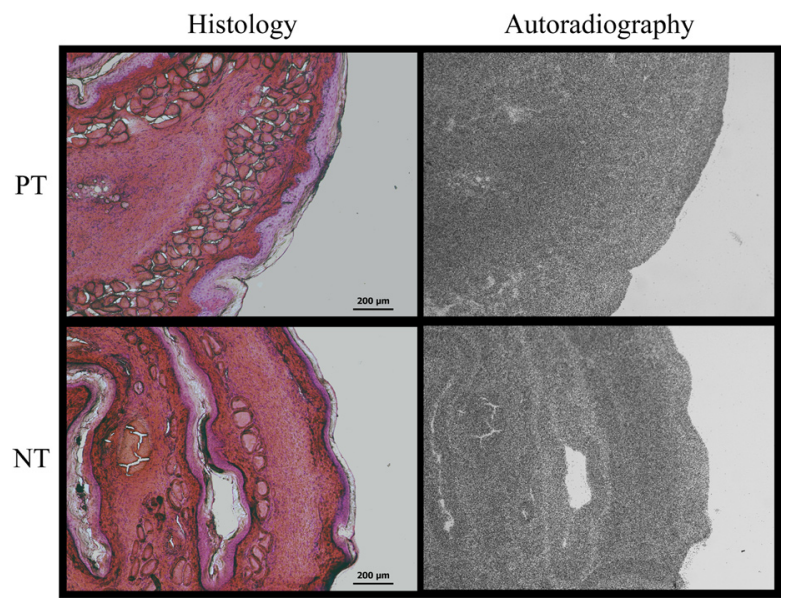

Figure 3. Light microscopy images of cryostat sections $(30 \mu \mathrm{m})$ stained with hematoxylin-eosin and the corresponding neutron autoradiography images $(10 \times) . \mathrm{PT}=$ premalignant tissue, $\mathrm{NT}=$ normal tissue. not pre-treated with thalidomide was statistically significant $(p=0.0233)$. Figure 3 shows that treatment with thalidomide and BPA-BNCT would render GB-10 distribution more homogeneous in precancerous tissue than in normal tissue. No changes in boron microdistribution were observed in normal tissue as a result of pre-treatment with thalidomide and BPA-BNCT.

\section{Discussion}

In the present study we combined two therapeutic modalities that have proved highly successful but failed to achieve response in $100 \%$ of the tumors when applied separately. Additionally, we can conclude that $\mathrm{Th}+\mathrm{Seq}-\mathrm{BNCT}$ is more effective than Th-Seq-BNCT because previously reported [11] tumor response data for Th-Seq-BNCT were compared with $\mathrm{Th}+$ Seq-BNCT (this study) and the differences reached statistical significance. The present irradiations were performed at the same neutron fluence as those in the previous study [11]. Briefly, the therapeutic efficacy of Seq-BNCT would result from three main features: 1) the use of two boron agents with different properties and complementary mechanisms of action, a strategy that contributes to boron targeting homogeneity; 2) the decrease in interstitial fluid pressure after the first part of the treatment that favors the subsequent distribution of blood-borne therapeutic agents, such as GB-10; and 3) the interval between BPA-BNCT and GB-10-BNCT that is short enough to preclude tumor cell repopulation and could favor re-targeting of the tumor cells that were refractory to the first application [11]. Aberrant blood vessel normalization prior to administration of BPA for BPA-BNCT would increase therapeutic efficacy by fixing the flawed delivery system. Herein, we demonstrated that aberrant blood vessel normalization prior to the administration of BPA favored BPA distribution although it did not increase absolute 
gross boron content in oral tumors. Temporary restoration of adequate blood flow would be enough to deliver BPA at therapeutically useful levels to otherwise inaccessible tumor areas [14].

The combined administration of aberrant blood vessel normalization and Seq-BNCT would profit from the benefits of both modalities. An additional advantage of the synergism between both modalities would be the increase in GB-10 boron targeting homogeneity induced by pre-treatment for aberrant blood vessel normalization followed by BPA-BNCT. This increase in targeting homogeneity for the second boron compound, GB-10, in addition to the described improvement in BPA distribution, would be pivotal to therapeutic efficacy. The neutron autoradiography studies reported herein stress the importance of boron microdistribution in BNCT efficacy and illustrate their potential contribution to protocol design. We have evidenced that it is possible to improve the efficacy of BNCT by modulating the distribution of the boron compounds currently authorized for use in patients. For BNCT to be successful, it is not enough to achieve tumor:blood and tumor:normal tissue boron concentration ratios of $\sim 3$ and absolute tumor boron concentration $>20$ ppm as classically described [4]. It is essential that the boron compounds should reach all the tumor cell subpopulations within the tumor. The results described herein suggest that addressing the issue of homogeneous boron targeting within tumor would contribute to pave the way for $\mathrm{BNCT}$ as a promising treatment modality. In particular, QTA was extremely contributory and allowed us to quantify the degree of homogeneity in boron targeting in terms of the $\mathrm{P} / \mathrm{S}$ ratio. The increase in targeting homogeneity of GB-10 in the Th+ GB-10 48 hours after BPA-BNCT group was statistically significant compared with ThGB-10 48 hours after BPA-BNCT. Based on previously reported data of therapeutic efficacy $[11,14]$ and the data reported herein, we propose a direct association between the percentage of tumor response and the degree of boron targeting homogeneity. Additionally, the apparent increase in boron targeting homogeneity in precancerous tissue might improve the previously described inhibitory effect of BNCT on the development of tumors from precancerous tissue [19].

Within the context of ongoing BNCT clinical trials for recurrent head and neck malignancies that showed encouraging tumor control associated to dose-limiting mucositis [3], the search for novel BNCT strategies that improve tumor control at no extra cost in terms of mucositis is particularly relevant. Within this context, Seq-BNCT preceded by aberrant blood vessel normalization would warrant cautious analysis in a clinical scenario. In this sense, the fact that GB-10 and BPA are both approved for use in humans is an additional advantage. Regarding the method employed for aberrant blood vessel normalization, thalidomide poses an advantage because it also exerts a protective effect on precancerous tissue mucositis [14]. Although thalidomide was withdrawn from the market due to its teratogenic effects, it was approved in 1997 by FDA to combat a variety of conditions [20]. However, other blood vessel normalization techniques in general [e.g. 21] that cannot be used in the hamster but are amenable to use in humans also merit investigation as a way of optimizing the therapeutic advantage of BNCT in patients.

\section{Acknowledgements}

The authors gratefully acknowledge the expert advice of Dr. Luis Otegui and the major statistical support provided by Dr. Gustavo Santa Cruz. The authors also acknowledge the generous gift of thalidomide by Triquim S.A. and Laboratorio Lazar (Argentina) and the expert support of Dr. Claudio Devida and his team with ICP-MS boron measurements. This study was supported in part by a grant from the National Agency for the Promotion of Science and Technology (ANPCyT) and a grant from the National Research Council (CONICET) of Argentina.

Declaration of interest: The authors report no conflicts of interest. The authors alone are responsible for the content and writing of the paper.

\section{References}

[1] Coderre JA, Morris GM. The radiation biology of boron neutron capture therapy. Radiat Res 1999;151:1-18.

[2] Kato I, Ono K, Sakurai Y, Ohmae M, Maruhashi A, Imahori Y, et al. Effectiveness of BNCT for recurrent head and neck malignancies. Appl Radiat Isot 2004;61: 1069-73.

[3] Kankaanranta L, Seppälä T, Koivunoro H, Saarilahti K, Atula T, Collan J, et al. Boron neutron capture therapy in the treatment of locally recurred head-and-neck cancer: Final analysis of a phase I/II trial. Int J Radiat Oncol Biol Phys 2012;82:e67-75.

[4] Barth RF, Vicente MG, Harling OK, Kiger WS 3rd, Riley KJ, Binns PJ, et al. Current status of boron neutron capture therapy of high grade gliomas and recurrent head and neck cancer. Radiat Oncol 2012;7:146.

[5] Barth RF. Boron neutron capture therapy at the crossroads: Challenges and opportunities. Appl Radiat Isot 2009;67(7-8 Suppl):S3-6.

[6] Coderre JA, Turcotte JC, Riley KJ, Binns PJ, Harling OK, Kiger WS 3rd. Boron neutron capture therapy: Cellular targeting of high linear energy transfer radiation. Technol Cancer Res Treat 2003;2:355-75.

[7] Kawabata S, Yang W, Barth RF, Wu G, Huo T, Binns PJ, et al. Convection enhanced delivery of carboranylporphyrins 
for neutron capture therapy of brain tumors. J Neurooncol 2011;103:175-85.

[8] Altieri S, Bortolussi S, Bruschi P, Chiari P, Fossati F, Stella $\mathrm{S}$, et al. Neutron autoradiography imaging of selective boron uptake in human metastatic tumours. Appl Radiat Isot 2008;66:1850-5.

[9] Portu A, Bernaola OA, Nievas S, Chiari P, Fossati F, Stella S. Measurement of $10 \mathrm{~B}$ concentration through autoradiography images in polycarbonate nuclear track detectors. Rad Meas 2011;46:1154-9.

[10] Portu A, Carpano M, Dagrosa A, Cabrini RL, Saint Martin G. Qualitative autoradiography with polycarbonate foils enables histological and track analyses on the same section. Biotech Histochem 2013;88:217-21.

[11] Molinari AJ, Pozzi EC, Monti Hughes A, Heber EM, Garabalino MA, Thorp SI, et al. "Sequential" boron neutron capture therapy (BNCT): A novel approach to BNCT for the treatment of oral cancer in the hamster cheek pouch model. Radiat Res 2011;175:463-72.

[12] Molinari AJ, Aromando RF, Itoiz ME, Garabalino MA, Monti Hughes A, Heber EM, et al. Blood vessel normalization in the hamster oral cancer model for experimental cancer therapy studies. Anticancer Res 2012;32:2703-9.

[13] Jain RK. Normalization of tumor vasculature: An emerging concept in antiangiogenic therapy. Science 2005;307:58-62.

[14] Molinari AJ, Pozzi EC, Monti Hughes A, Heber EM, Garabalino MA, Thorp SI, et al. Tumor blood vessel "normalization" improves the therapeutic efficacy of boron neutron capture therapy (BNCT) in experimental oral cancer. Radiat Res 2012;177:59-68.

[15] Miller M, Quintana J, Ojeda J, Langan S, Thorp S, Pozzi E, et al. New irradiation facility for biomedical applications at the RA-3 reactor thermal column. Appl Radiat Isot 2009; 67(7-8 Suppl):S226-9.

[16] Pozzi E, Nigg DW, Miller M, Thorp SI, Heber EM, Zarza $\mathrm{L}$, et al. Dosimetry and radiobiology at the new RA-3 reactor boron neutron capture therapy (BNCT) facility: Application to the treatment of experimental oral cancer. Appl Radiat Isot 2009;67(7-8 Suppl):S309-12.

[17] López-Castaño F, Oñate-Sánchez RE, Roldán-Chicano R, Cabrerizo-Merino MC. Measurement of secondary mucositis to oncohematologic treatment by means of different scale. Med Oral Patol Oral Cir Bucal 2005;10:412-21.

[18] Sonis ST, Peterson RL, Edwards LJ, Lucey CA, Wang L, Mason L, et al. Defining mechanisms of action of interleukin-11 on the progression of radiation-induced oral mucositis in hamsters. Oral Oncol 2000;36:373-81.

[19] Monti Hughes A, Pozzi E, Thorp S, Garabalino M, Farías R, González S, et al. Boron neutron capture therapy for oral precancer: Proof of principle in an experimental animal model. Oral Dis 2013;19:789-95.

[20] Neiger BL. The re-emergence of thalidomide: Results of a scientific conference. Teratology 2000;62:432-5.

[21] Strickler JH, Hurwitz HI. Bevacizumab-based therapies in the first-line treatment of metastatic colorectal cancer. Oncologist 2012;17:513-24. 